\title{
Suppression of SLC11A2 Expression Is Essential to Maintain Duodenal Integrity During Dietary Iron Overload
}

Tomoyuki Shirase, ${ }^{*}$ Kiyoshi Mori, ${ }^{\dagger}$

Yasumasa Okazaki, ${ }^{\ddagger}$ Ken Itoh, ${ }^{\S}$

Masayuki Yamamoto, " Mitsuaki Tabuchi," Fumio Kishi, ${ }^{,}$Li Jiang, ${ }^{* \neq}$ Shinya Akatsuka, ${ }^{* \neq}$ Kazuwa Nakao, ${ }^{\dagger}$ and Shinya Toyokuni ${ }^{\star \ddagger}$

From the Departments of Pathology and Biology of Diseases," and Medicine and Clinical Science, ${ }^{\dagger}$ Kyoto University Graduate School of Medicine, Kyoto; the Department of Pathology and Biological Responses, ${ }^{\ddagger}$ Nagoya University Graduate School of Medicine, Nagoya; the Center for Advanced Medical Research, ${ }^{\S}$ Hirosaki University Graduate School of Medicine, Hirosaki; the Department of Medical Biochemistry, "Tohoku University Graduate School of Medicine, Sendai; and the Department of Molecular Genetics," Kawasaki Medical School, Kurashiki, Japan

Iron is essential for the survival of mammals, but iron overload causes fibrosis and carcinogenesis. Reduced iron absorption and regulated release into circulation in duodenal mucosa constitute two major mechanisms of protection against dietary iron overload; however, their relative contribution remains elusive. To study the significance of the former process, we generated SLC11A2 transgenic mice (TGs) under the control of the chicken $\beta$-actin promoter. TGs were viable and fertile, and displayed no overt abnormalities up to 20 months. No significant difference in iron concentration was observed in major solid organs between TGs and their wild-type littermates, suggesting that increased number of iron transporters does not lead to increased iron absorption. To test the sensitivity to iron overload, TGs and wild-type mice were fed with an iron-rich diet containing $2 \%$ ferric citrate. Iron supplementation caused suppression of endogenous duodenal SLC11A2 expression, downregulation of duodenal ferroportin, and overexpression of hepatic hepcidin, precluding excessive iron uptake both in the TGs and wild-type mice. However, iron-treated TGs revealed increased mortality, resulting from oxidative mucosal damage leading to hemorrhagic erosion throughout the whole intestinal area. These findings suggest that reduced iron release from duodenal cells into circulation plays a role in mitigating excessive iron uptake from the diet and that finely regulated duodenal absorption is essential to protect intestinal mucosa from iron-induced oxidative damage. (Am J Pathol 2010, 177:677-685; DOI: 10.2353/ajpath.2010.090823)

Iron is one of the most abundant metals on earth and is crucial for all kinds of life. Iron is an essential component of hemoglobin, myoglobin (ie, in the transport and utilization of oxygen), and heme enzymes such as cytochromes, catalase, and peroxidases. Intricate regulatory mechanisms have evolved to preserve iron even in an iron-deficient environment. Thus, there is no regulated iron excretion in mammals through bile or urine; instead, iron leaves the body only through bleeding or sloughing off of the skin or mucosal cells. Surplus iron is generally stored in hepatocytes and macrophages. ${ }^{1,2}$ When a tissue's storage capacity is persistently exceeded, unsequestered iron catalyzes the formation of reactive oxygen species, which leads not only to tissue fibrosis with clinical manifestations, such as cirrhosis, cardiomyopathy, and endocrinopathies, but also to a high risk of cancer. ${ }^{3,4}$

Iron cannot pass through plasma membranes without a specific transporter. To date, only a single transmembrane transporter, solute carrier family 11, member 2 (SLC11A2; also known as divalent metal ion transporter 1 [DMT1], NRAMP2, and DCT1) is known to have physiological importance in bringing iron into cells. SLC11A2 acts as a proton-coupled iron importer of $\mathrm{Fe}^{2+} .5$ It can

Supported in part by a Ministry of Education, Culture, Sports, Science and Technology of Japan grant (Special Coordination Funds for Promoting Science and Technology), a grant from Takeda Science Foundation, a Grant-in-Aid for Cancer Research from the Ministry of Health, Labor and Welfare of Japan, and a Grant-in-Aid from the Ministry of Education, Culture, Sports, Science and Technology of Japan.

Accepted for publication April 2, 2010.

Address reprint requests to Shinya Toyokuni, MD., Ph.D., Department of Pathology and Biological Responses, Nagoya University Graduate School of Medicine, 65 Tsurumai-cho, Showa-ku, Nagoya, Aichi 4668550, Japan. E-mail: toyokuni@med.nagoya-u.ac.jp. 
also transport a variety of other divalent metal cations, including $\mathrm{Mn}^{2+}, \mathrm{Co}^{2+}, \mathrm{Cu}^{2+}$, and $\mathrm{Zn}^{2+}, 5$ although iron appears to be its most important physiological substrate. SLC11A2 is located on the apical membrane of duodenal surface cells, consistent with its role in transepithelial iron transport. ${ }^{6}$ SLC11A2 is also located in transferrin-cycle endosomes, ${ }^{2,7}$ where it participates in iron transfer to the cytoplasm. Additionally, it is present in hepatocytes, where it has been postulated to be involved in nontransferrin-bound iron uptake. ${ }^{8}$

Studies of animals carrying missense mutations in SLC11A2 have demonstrated two major roles for this transporter: intestinal nonheme iron transport and iron uptake especially in erythroid cells. Microcytic anemia $(m k)$ mice and Belgrade (b) rats present systemic iron deficiency and anemia attributable to the same spontaneous missense mutation (G185R) in SLC11A2. ${ }^{9,10}$ Recently, cases of human SLC11A2 mutation have also been reported. The patients revealed marked anemia, but in contrast to rodents with SLC11A2 mutations, hepatic iron overload was observed at early ages. ${ }^{11,12}$ Moreover, a murine model of systemic inactivation of SLC11A2 has been generated, resulting in a phenotype that is more severe than that seen in animals homozygous for the G185R mutation. Although SLC11A2 knockout mice were born alive, they showed progressive postnatal growth retardation, and all mice died before the seventh day. ${ }^{13}$ In the present study, by generating SLC11A2 transgenic mice under the control of the $\beta$-actin promoter, we tested a hypothesis that SLC11A2 overexpression would accumulate iron in various organs.

\section{Materials and Methods}

\section{Cell Culture and Chemicals}

COS-7 cells (American Type Culture Collection, Manassas, VA) were cultured in Dulbecco's modified Eagle's medium (GIBCO, Invitrogen Life Technologies, Carlsbad, CA) supplemented with $10 \%$ fetal bovine serum, 100 $\mathrm{U} / \mathrm{ml}$ penicillin, $100 \mu \mathrm{g} / \mathrm{ml}$ streptomycin, and $2 \mathrm{mmol} / \mathrm{L}$ L-glutamine at $37^{\circ} \mathrm{C}$ and $5 \% \mathrm{CO}_{2}$. Transfection was performed with Lipofectamine 2000 (Invitrogen) according to the supplier's protocol. All of the chemicals used were of analytical grade.

\section{Transgenic Mice}

A part of the rat SLC11A2 (rSLC11A2) CDNA, including the full-length protein coding region and the following 750 bp of 3'-UTR (IRE + ) region (GenBank accession number AF008439; exon 1B, $\left.{ }^{14} \mathrm{DMT}_{1} \mathrm{~A}^{15}\right)$, was inserted into the pCAGGS-green fluorescent protein (GFP) vector (GenBank accession number BD178301), which carries a chicken $\beta$-actin promoter. This vector was a gift from Dr. Junichi Miyazaki (Osaka University, Japan). The vector's GFP sequences were replaced by the rSLC11A2 cDNA. The 4.7-kb transgene was cut out of the vector by Sall and Hindlll digestion, purified, and used to generate transgenic mice. The pronuclei of fertilized eggs from hyperovulated C57BL/6 crossed with DBA/2 were microinjected with this DNA construct. Three lines of transgenic mice were established (rDMT-e, rDMT-n, and rDMT-q; deposited in RIKEN BioResource Center, Tsukuba, Ibaraki, Japan), and all three of the lines were used for further analyses. Genotype was determined by PCR by using tail genomic DNA. The transgene was amplified by using the forward primer 5'-GACAAGGGTTTCTTCCTTGTTGTCCTGG-3' (annealing to the $3^{\prime}$-UTR region of rSLC11A2) and the reverse primer 5'-TTTGCCCTCCCATATGTCCTTCCGAG-3' (annealing to the vector's sequence following the $3^{\prime}$-UTR region). The expression of TG-derived transcripts was also confirmed by reverse transcription-PCR analysis. The animals were bred under specific pathogen-free conditions and an AIN93M diet (Funabashi Farms Co., Ltd., Chiba, Japan). The animal research committees of Kyoto University Graduate School of Medicine and of Nagoya University Graduate School of Medicine approved this experiment. The animals were maintained in a heterozygous state of the transgene. The animals were fasted for more than 15 hours and euthanized between 9 AM and noon.

\section{Southern Blot Analysis}

For the evaluation of transgene copy number, $20 \mu \mathrm{g}$ of tail DNA was digested with EcoRI, separated on $1.0 \%$ agarose gel, and transferred onto a Hybond-N membrane (Amersham Biosciences, Buckinghamshire, UK).

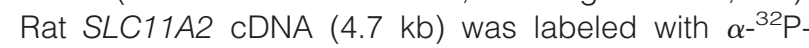
dCTP (Amersham) and used as a probe for Southern blot analysis. The membrane was exposed to an X-ray film (Kodak, Rochester, NY) as previously described, ${ }^{16}$ which generated $2.4 \mathrm{~kb}$ band corresponding to transgene.

\section{Inverse PCR Analysis}

Inverse PCR analysis was done as described ${ }^{17}$ to locate the $3^{\prime}$ ends of transgene in the genome of each line. Genomic DNA was digested with Haelll and self-ligated with T4 ligase. Adjacent endogenous genomic DNA fragments were amplified by PCR with a pair of primers prepared within the transgene (forward primer, F-45055'-GCTGTCCATTCCTTATTCCATAGAAA-3'-4530 and reverse primer, R-4312-5'-TTTATTAGCCAGAAGTCAGATGCTCA-3'-4287), cloned, sequenced, and matched with the public database (http://genome.ucsc.edu, last accessed January 31, 2010).

\section{Antibodies}

An anti-rat SLC11A2 rabbit polyclonal antibody was produced by a commercial supplier (Hokudo, Hokkaido, Japan). Briefly, a 13-mer oligopeptide ( $\mathrm{NH}_{3}$-CVLLSEDTSGGNTK-COOH) corresponding to the amino acids 549561 of the rat SLC11A2 with an additional cysteine was synthesized and conjugated to keyhole limpet hemocyanin, which was used as an immunogen for JW rabbits. One week after the third immunization, whole serum was harvested and purified by using a SulfoLink kit with the 
same oligopeptide (Pierce, Rockford, IL). Mouse monoclonal antibodies against 8-hydroxy-2'-deoxyguanosine $(8-\mathrm{OHdG})^{18}$ and 4-hydroxy-2-nonenal (HNE)-modified proteins ${ }^{19}$ were produced in our laboratory and used to locate oxidative stress ${ }^{20}$ as previously described. ${ }^{21}$

\section{Western Blot Analysis}

Male 6-week-old mice were used for the analysis. Preparation of the samples and Western blot analysis were performed as previously described ${ }^{22,23}$ with modifications. For intestinal samples, mucosa was scraped off with razor blade following wash with physiological saline solution (PSS). The mucosal samples were directly put into Laemni sample buffer, sheard with a 25-Gauge needle, heated at $95^{\circ} \mathrm{C}$ for 6 minutes, and used as samples for electrophresis. For tissues other than intestine, samples in Laemni buffer were heated at $60^{\circ} \mathrm{C}$ for 15 minutes. Deglycosylation of proteins was done with PNGase $\mathrm{F}$ (New England Biolabs, Ipswich, MA) as previously described. ${ }^{24}$ The working concentration of the SLC11A2 antibody was $1.25 \mu \mathrm{g} / \mathrm{ml}$. Horseradish peroxidase-conjugated monoclonal antibody for $\beta$-actin (mAbcam 8226, Abcam, Cambridge, UK) was used for the loading control of Western blot analysis. Dot blots were performed in a similar fashion except that electrophresis and membrane transfer were not done.

\section{Histological and Immunohistochemical Analysis}

For routine microscopic analysis, hematoxylin and eosin staining was used following 10\% phosphate-buffered formalin fixation and paraffin embedding. Immunohistochemistry was performed as previously described. ${ }^{25}$ The working concentration of the SLC11A2 antibody used was $5 \mu \mathrm{g} / \mathrm{ml}$. Control sections were treated without primary antibody, and all of the control sections remained free of immunostaining. Quantitation was performed as previously described. ${ }^{18}$

\section{RT-PCR Analysis}

To quantify mRNA levels, total RNA was extracted by means of a modified acid guanidinium phenol chloroform method (Isogen, Nippon Gene, Tokyo). One microgram of total RNA was reverse-transcribed with a first-strand cDNA synthesis kit (GE Health care, Buckinghamshire, UK) following the manufacturer's instructions, and was used as template for PCR amplification of rSLC11A2, mouse SLC11A2 (mSLC11A2), SLC4OA1 (ferroportin-1), HAMP (hepcidin microbial peptide), TFRC (transferrin receptor), and GAPDH genes. For quantitative real-time PCR, a Platinum SYBR Green qPCR SuperMix UDG kit (Invitrogen) and real-time PCR system 7300 (Applied Biosystems, Foster City, CA) were used. Primer sequences used were as follows: GAPDH, forward primer F-5'-AACTTTGGCATTGTGGAAGG-3' and reverse primer R-5'-CACATTGGGGGTAGGAACAC-3'; rSLC11A2, F-5'-TGCTTGGTGGCCTAAAACTC-3' and R-5'-CCCCTGACAAAACCAGTCAT-3'; and $m S L C 11 A 2, F-5$ '-TGTTTTTGTGAAATAGCATCT-
TGC-3' and R-5'-GACCCCCAACAAAACTCATC-3' (based on GenBank accession number AK049856.1). The primer pairs used for mouse ferroportin-1 and hepcidin, ${ }^{26}$ and for mouse transferrin receptor, ${ }^{27}$ were as previously described.

\section{Tissue Iron Measurements}

Tissue iron concentrations were determined with a Z-7000 polarized Zeeman atomic absorption spectrometer (Hitachi, Ltd., Tokyo, Japan) as previously described. ${ }^{28}$

\section{Iron Uptake Study}

Two centimeters of duodenum was dissected, and the accompanying mesentery was removed. Both ends were ligated with 6-0 clear nylon threads. An aliquot (100 $\mu$ l) of PSS containing $\mathrm{FeSO}_{4}(200 \mu \mathrm{mol} / \mathrm{L}, \mathrm{pH}$ 6.2) and ${ }^{55} \mathrm{FeCl}_{3}(1 \mu \mathrm{mol} / \mathrm{L}$, PerkinElmer, Boston, MA) was injected into the lumen and incubated for 10 minutes at $37^{\circ} \mathrm{C}$. The reaction was terminated by opening the lumen in PSS, and the duodenum was washed vigorously three times (5 minutes each) with ample PSS containing $2000 \mu \mathrm{mol} / \mathrm{L}$ $\mathrm{FeSO}_{4}$. The duodenal samples were lysed in $100 \mu$ l of $2 \%$ sodium dodecyl sulfate solution and ${ }^{55} \mathrm{Fe}$ incorporated was measured with LS 6000 liquid scintillation counter (Beckman Coulter Inc., Fullerton, CA). In these experiments, $1 \%$ to $5 \%$ of iron out of 20,000 pmole was taken into the duodenal mucosa within 10 minutes.

\section{Iron-Rich Diet Study}

Adult mice (6 to 7 weeks old) were kept under a high-iron diet for 4 weeks. Diets were formulated by AIN-93M, supplemented with $2 \%(\mathrm{w} / \mathrm{w})$ ferric citrate (Sigma, St. Louis, MO).

\section{Statistical Analysis}

Statistical analyses were performed with one-way analysis of variance and an unpaired $t$-test. Differences in survival were assessed by Kaplan-Meiyer methods and analyzed by both a generalized Wilcoxon test and Log rank test.

\section{Results}

\section{Functional Analysis of Transgenic Expression Vector}

A rat SLC11A2 cDNA (IRE ${ }^{+}$) under the control of chicken $\beta$-actin promoter was used to generate transgenic mice. COS-7 cells were transfected with the transgenic expression vector. Intense four bands of $\sim 50$ or $\sim 100 \mathrm{kDa}$, two each, respectively, with smear at $>100 \mathrm{kDa}$ appeared after transfection of rSLC11A2 vector, but not of GFP vector, by Western blot analysis (Figure 1A). Deglycosylation caused the decrease in $\sim 100 \mathrm{kDa}$ bands with increase in the lower $\sim 50 \mathrm{kDa}$ band (Figure 1B). Immunostaining of the cells for rat SLC11A2 24 hours after 
A

$$
\text { 造 }
$$

B

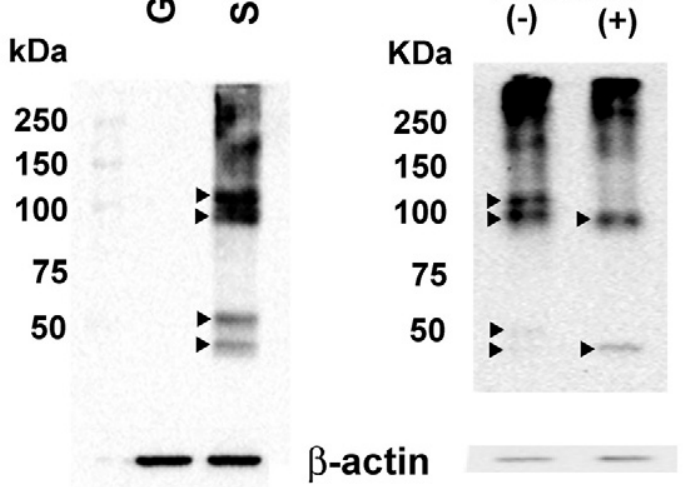

\section{C}
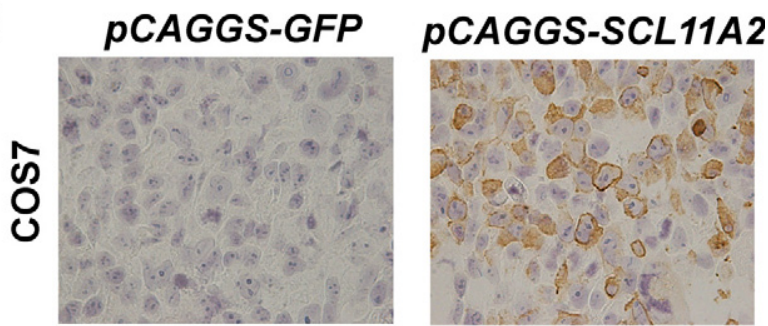

\section{D}

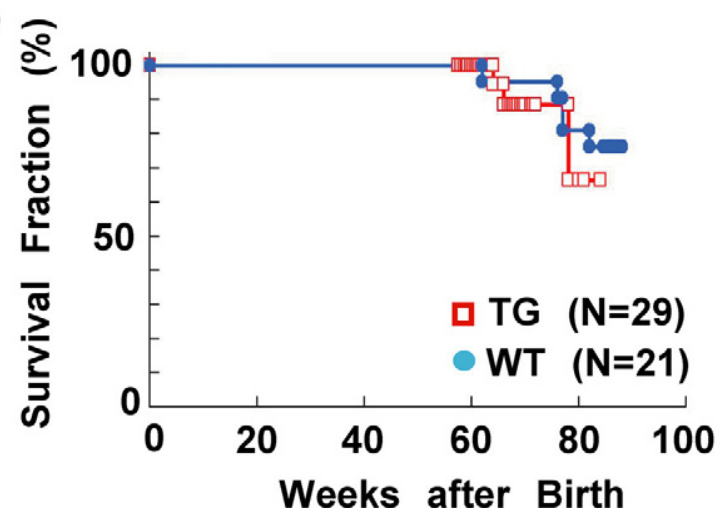

Figure 1. SLC11A2 expression vector and generation of transgenic mice. A: Western blot analysis of rat SLC11A2 expression after transfection of the constructed expression vector into COS7 cells. An anti-rat SLC11A2 antibody recognizing a C-terminal polypeptide was applied as described in Materials and Methods. Four main bands (arrowheads) and high-molecular weight smear appeared. Transfection of GFP-construct presented no signal. B: Glycosylation of SLC11A2. Deglycosylation by PNGase F decreased the intensity of the bands (arrowheads), but intensified the lower $\sim 50 \mathrm{kDa}$ band. Refer to text for discussion. C: Immunohistochemical analysis of COS7 cells after SLC11A2 transfection. Cell block was used with hematoxylin nuclear counterstaining. Immunopositivity is observed both in the cytoplasm and plasma membrane. D: Survival curve of TG and WT. No significant difference was observed under a basal diet.

transfection revealed that approximately $50 \%$ of cells expressed rat SLC11A2, and the localization was both at the plasma membrane and cytoplasm (Figure 1C). Cellular iron concentration doubled compared with the mock transfection control cells $\left(1.00 \mu \mathrm{g} \mathrm{Fe} / 10^{6}\right.$ cells vs. 0.52 $\mu \mathrm{g} \mathrm{Fe} / 10^{6}$ cells; means of duplicate study). These results confirmed the quality of both the expression vector and the affinity-purified antibody.

\section{Characterization of SLC11A2 Transgenic Mice}

Three lines of transgenic mice (TGs) were established (rDMT-e, rDMT-n, and rDMT-q). Each line of TGs was back-crossed to C57BL/6 for more than 10 generations to minimize the DBA background, and these mice were used for the following studies. Dot blot analysis of tail protein was useful for genotyping and completely matched with the results by PCR analysis (data not shown).

The TGs were observed up to 85 weeks of age, and no apparent macroscopic difference in appearance or behavior was observed (Figure 1D). Histopathological analysis also revealed no detectable differences in each organ in the TG. This was common among all three of the established lines. At the same time, we undertook to identify the genomic location of the transgene to rule out the possibility of insertional mutagenesis. Southern blot analysis revealed that the copy number of inserted transgene in each line was within a few copies (data not shown). Inverse PCR analysis identified the loci of transgene insertion in two of the lines (e-strain, within Line-1 repeat sequence; $n$-line, not identified probably due to tandem repeat of transgene; q-line, chromosome 6q-ter, 147,068,000-147,068,200; 37 kbp upstream of exon 1 for Ppfibp1 gene).

Western blot analysis of TG revealed an intense but broad band of $\sim 100 \mathrm{kDa}$ in the lung and liver, and weaker bands of similar molecular weight in the heart and testis. In the duodenum, TGs showed a sharp but weak band of $\sim 50 \mathrm{kDa}$ with an intense band at $\sim 30 \mathrm{kDa}$ (Figure 2A), whereas the wild-type mice (WTs) showed faint bands at $50 \mathrm{kDa}$. Solid organs (cerebrum, cerebellum, heart, lung, spleen, liver, kidney, and testis) of WTs presented no bands (data not shown). These differences in organs might be attributed not only to the glycosylation or proteolysis of SLC11A2 but also the structural difficulty as membrane protein. In contrast, immunohistochemical studies revealed high levels of rSLC11A2 in a variety of TG organs, including the duodenum (apical surface cells), renal proximal tubules, lung (apical surface of bronchial cells), liver (Kupffer cells and bile ducts), stomach (fundic gland chief cells and squamous cells of forestomach), colonic epithelial cells, and Sertoli and Leydig cells (Figure 2B), as compared with the corresponding organs of wild-type in which only the duodenal villi showed recognizable expression, but the other organs had a limited and faint staining. Hepatocytes of TGs did not show high expression of SLC11A2 (Figure 2B).

The total level (mouse and rat) of SLC11A2 mRNA expression in the TG duodenum was higher than that of wild-type although the endogenous murine SLC11A2 mRNA level was suppressed (Figure 3). The same tendency for mRNA was observed in the liver as a whole (70\% increase of total SLC11A2 mRNA with 25\% endogenous share).

\section{Iron Concentration in Transgenic Mice}

The iron concentration in various organs including heart, lung, spleen, liver, kidney, brain, and testis of mice (TGs 


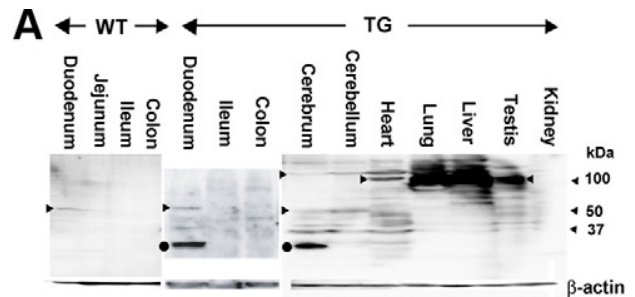

Figure 2. Protein levels of SLC11A2 transgenic mice. A: Western blot analysis of SLC11A2. Arrowheads show the position of recognized major bands. Scraped duodenal mucosa and lysates from cerebrum, heart, lung, liver, and testis in TG showed intense bands, although the bands appeared at different molecular weights with or without smear. Duodenal mucosa from WT also showed a faint band (arrowhead). There was much difficulty in this procedure because of the presence of 12 membrane-spanning domains in SLC11A2. Closed circle, possible proteolytic product. Refer to Materials and Methods and Discussion sections for details. B: Immunohistochemical analysis of SLC11A2. Many kinds of parenchymal cells showed positivity. Immunostaining was most intense at the apical membrane of the duodenum Kupffer cells and bile ducts, but not hepatocytes, showed positivity. Refer to the Results section for details (Scale bar $=$ $100 \mu \mathrm{m}$ in organs other than lung and testis; Scale bar $=200$ $\mu \mathrm{m}$ in lung; and Scale bar $=50 \mu \mathrm{m}$ in the duodenal inset and testis).
B
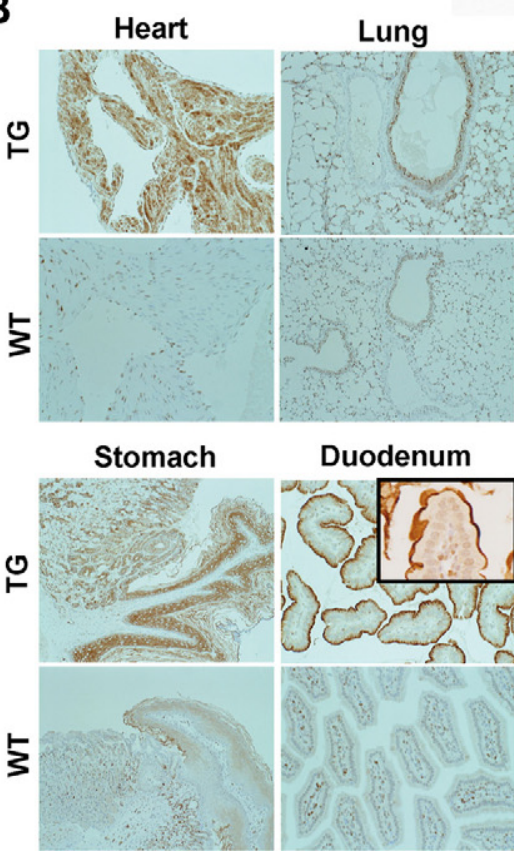

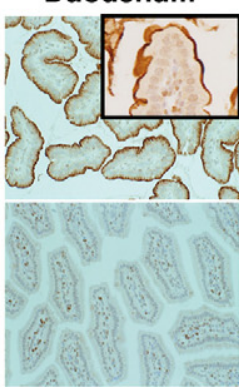

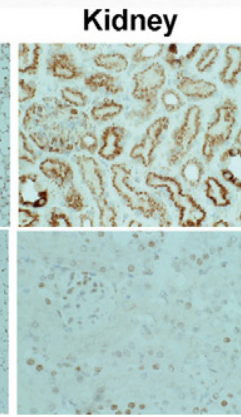

Colon

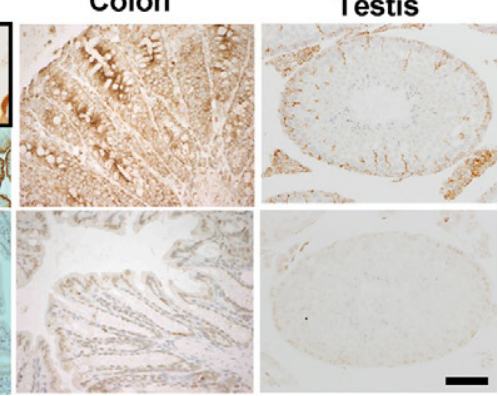

Liver and wild-type mice of 18-week-old males; $N=4$ ) fed with basal diet was measured. There was no significant difference in iron concentration between wild-type mice and TGs (data not shown). No iron deposits were observed in the duodenum with Perls' iron staining under a basal diet. The serum iron concentration was also not significantly

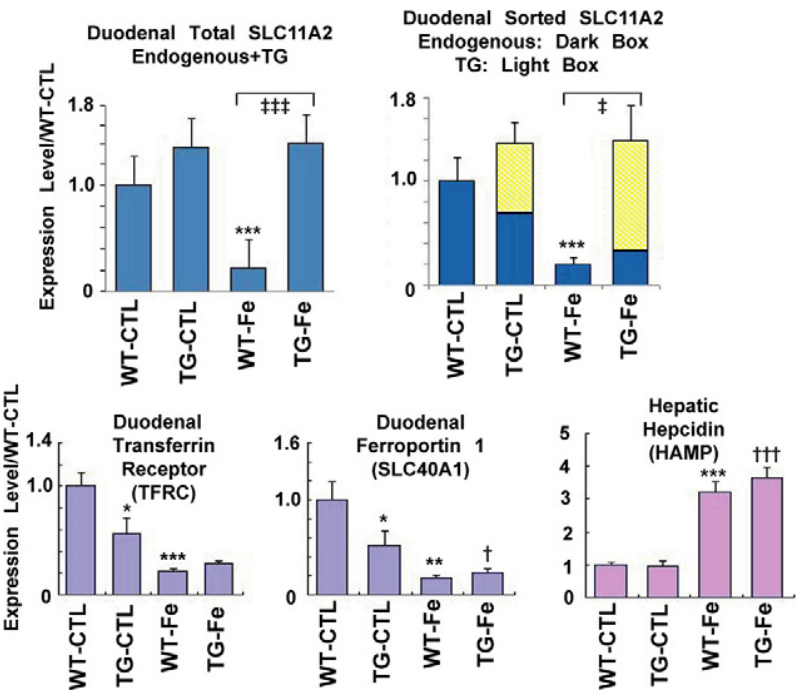

Figure 3. Iron metabolism in the SLC11A2 transgenic mice. Expression of iron metabolism genes before and after an iron-rich diet ( 24 hours after start). The overall mRNA expression of SLC11A2 was constantly higher in the transgenic mice, which was especially prominent under an iron-rich diet. An iron-rich diet suppressed the duodenal expression of the transferrin receptor and ferroportin-1 genes, but increased the hepatic expression of hepcidin $\left(N=4\right.$ to 5 , means \pm SEM; ${ }^{*} P<0.05 ;{ }^{* *} P<0.01 ;{ }^{* * * *} P<0.001$ vs. WT-CTL; ${ }^{\dagger} P<0.05 ;{ }^{\dagger+t} P<0.001$ vs. TG-CTL; ${ }^{\ddagger} P<0.01 ;{ }^{\# \# P} P<0.001$ vs. WT-Fe). One-way analysis of variance test for comparison among multiple groups detected statistical significance in all of the analyses of five genes (Total SLC11A2, $P=0.0034$; Endogenous SLC11A2, $P=0.0012 ;$ TFRC, $P=0.0007$; SLC4OA1, $P=0.0020$; and HAMP, $P=0.00002$ ). CTL, control basal diet; WT-Fe, wild-type mice under an iron-rich diet; TG-Fe, transgenic mice under an iron-rich diet. different between TGs and wild-type mice under a basal diet (data not shown).

\section{Iron Uptake Study}

To evaluate the difference in the duodenal iron absorption, the dissected duodenum was ligated at both ends, ${ }^{55} \mathrm{Fe}$ was injected into the lumen, and the remaining radioisotope activity in the duodenal wall was measured after vigorous washings. ${ }^{55} \mathrm{Fe}$ uptake was significantly higher in the TGs (TG: $12.8 \pm 1.9 \mathrm{pmol} \mathrm{Fe} / \mathrm{mg}$ wet weight/10 minutes versus wild-type: $7.1 \pm 2.1 \mathrm{pmol}$ $\mathrm{Fe} / \mathrm{mg}$ wet weight/10 minutes; means $\pm \mathrm{SEM}, N=4 ; P<$ $0.05)$, demonstrating that the transgene is functional.

\section{Iron Metabolism in Transgenic Mice}

Based on the obtained results, we hypothesized that the iron absorption by duodenal mucosal cells is blocked and does not enter into the portal circulation. To test this hypothesis, we measured the duodenal mRNA levels of ferroportin-1 (SLC40A1), the secreting transporter from duodenal cells and those of transferrin receptor (TFRC) under a basal diet. Both of the mRNA levels were significantly decreased in the TGs despite steady levels of hepatic hepcidin mRNA (Figure 3), confirming that the idea of a "mucosal block" that provides a feedback mechanism for iron absorption is functional. Then, we performed an iron-rich diet study.

\section{Iron-Rich Diet Study}

Male mice (6 to 7 weeks old) of both TG and wild-type background were fed with an iron-rich diet (TG, $N=46$; wild-type, $N=51$ ). This diet caused marked weight loss 

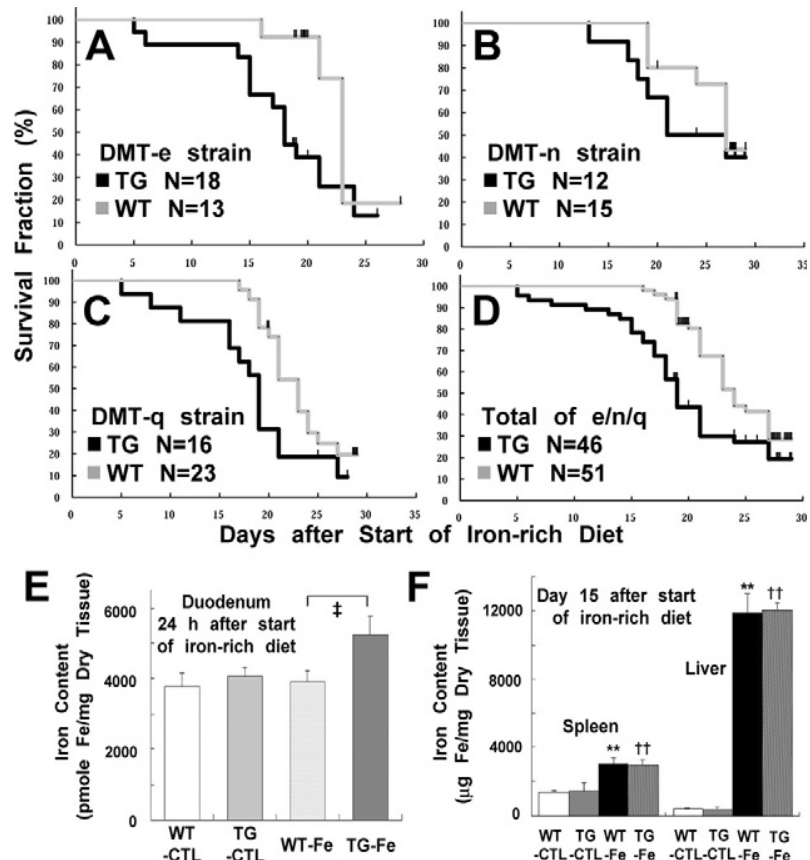

Figure 4. Effects of an iron-rich diet in the SLC11A2 transgenic mice. A-C Survival curve of each strain. TGs and WT mice were compared with littermates. SLC11A2 transgenic mice were more susceptible to an iron-rich diet (rDMT-e: $P<0.05$; rDMT-n: $P=0.26$ at day $15, P=0.34$ at day $20, P=0.18$ at day 25 , and $P=0.46$ at day 29; rDMT-q: $P<0.05)$. D: Survival curve of the combined three strains $(P<0.01)$. E: Duodenal iron content under an iron-rich diet. Iron content was significantly increased in the duodenum of TG 24 hours after the start of an iron-rich diet $(N=5$, means \pm SEM; analysis of variance, $P=0.087 ;{ }^{\ddagger} P<0.05$ vs. WT-Fe). F: Iron content in the spleen and liver under an iron-rich diet. Iron content in the spleen and liver was significantly increased, demonstrating that iron absorption was significantly increased under an iron-rich diet despite a feedback mechanism in the duodenum ( $N=4$ to 5 , means \pm SEM; analysis of variance, $P=0.0017$ for spleen and $P=4.5 \times 10^{-9}$ for liver; ${ }^{* *} P<0.01$ vs. WT-CTL; ${ }^{+\dagger} P<0.01$ vs. TG-CTL).

with melena within a week in both groups, and most of the mice died within 4 weeks. All of the three lines showed the same tendency, with rDMT-n line milder phenotype (Figure 4, A-D). Then, we focused on the early period of up to day 20 , where the number of survivors in both groups exceeded $50 \%$. In the TG group, the first mouse died at day 5 and the survival rate dropped to $75 \%$ at day 16, when all 51 of the WTs were still alive (Figure 4D). This study showed a significant survival difference between the two groups $(P<0.0001)$. At day 19 , the survival rate of TGs was $56 \%$, whereas that of the wild-type group was $90 \%$. The iron concentration in the duodenum was measured 24 hours after the start of the iron-rich diet and in various organs at day 15 . This study showed a higher iron content in the duodenum at day 1 (Figure 4E), but no significant iron increase in the heart, lung, kidney, brain, and testis was observed at day 15 (data not shown). Notably, a significant increase was observed in the liver and spleen, but with no statistical difference between the two groups (Figure 4F).

\section{Causes of Death in the Iron-Rich Diet Study}

All of the mice in this experiment showed marked weight loss (20\% to $30 \%$ ), severe anemia (hematocrit of 10 to
A

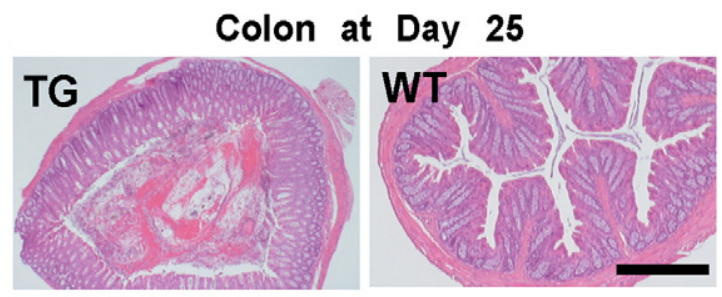

B

Duodenum at Day 2

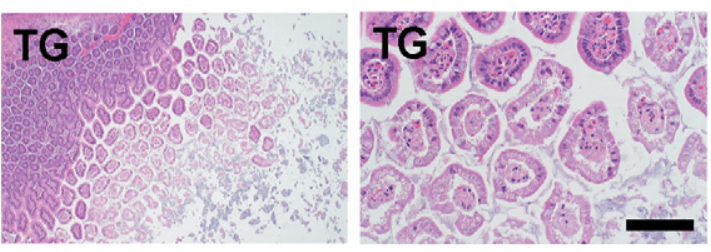

C

Duodenum at Day 5
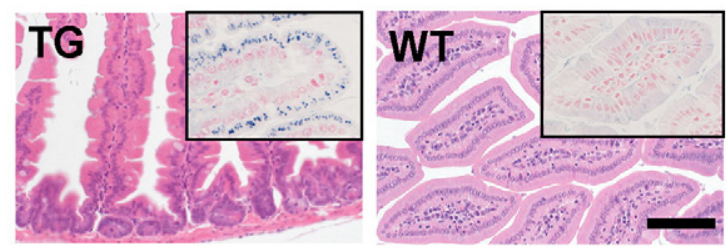

D

Duodenum at Day 5
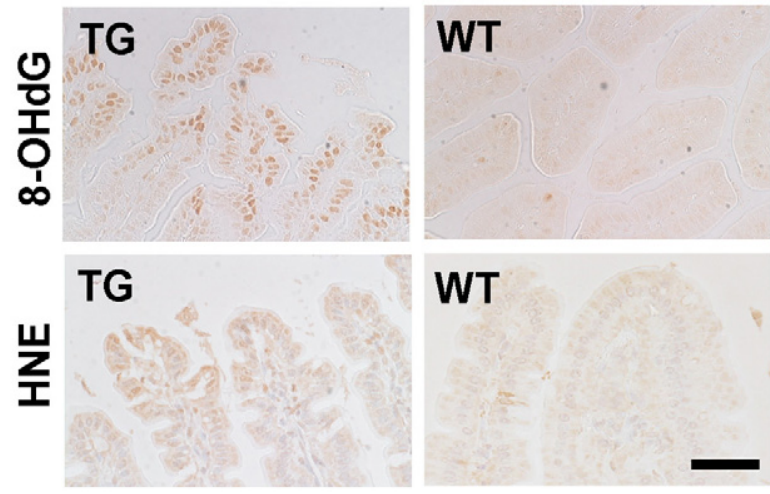

\section{WT}

Figure 5. Pathology of the intestine in SLC11A2 transgenic mice under an iron-rich diet. A: Low-power view of colon at day 25 after the start of an iron-rich diet. TGs revealed hemorrhagic erosion, whereas WT mice showed normal histology (Scale bar $=500 \mu \mathrm{m})$. B: Duodenum at day two of TG after the start of an iron-rich diet. Erosion of the apical surface of the duodenal epithelial cells was prominent (Scale bar $=400 \mu \mathrm{m}$ and $100 \mu \mathrm{m}$, respectively). C: Iron deposits in the duodenal cells of survived mice at day five. Degenerative duodenal cells in TG accumulate iron in the cytoplasm, whereas those in wild-type do not (Scale bar $=140 \mu \mathrm{m})$. Inset, Perls' iron staining (Scale bar $=70 \mu \mathrm{m})$. D: Oxidative stress in the duodenal cells at day five. Increase in nuclear 8-hydroxy-2'-deoxyguanosine (8-OHdG) and cytoplasmic 4-hydroxy-2-nonenal (HNE)-modified proteins were increased, indicating the pathological process is oxidative stress-dependent at day five (Scale bar $=70 \mu \mathrm{m})$.

15), and coagulation attaching around the perianal region. In addition, postmortem examination showed severe gastrointestinal bleeding, especially from the large intestine (Figure 5A), and a marked atrophic change of the whole small intestine. All of these findings strongly suggested that a prolonged and uncontrollable gastrointestinal ulcer and erosion by the high amounts of iron were the main pathological problems, and that continuous gastrointestinal blood loss caused dehydration, severe anemia, and malnutrition, finally leading to death. 
The severity of pathological findings was higher in the TGs than wild-type mice.

\section{Early Histological Alterations Under an Iron-Rich Diet}

To confirm this observation, the day 2 mice were sacrificed and the organs were examined. In most of the cases, the middle to distal portions of the small intestine were segmentally distended and presented with a translucent wall appearance. On microscopic observation, marked degeneration and necrosis of the villous structure was observed throughout the small intestine. Mucinous material originating from the degeneration and necrosis filled the bowel (Figure 5B). At day 5, the distention seen at day 2 was no longer prominent; instead, most of the small intestine showed a marked atrophic change, which was thought to be the consequence of a loss of the villi. At day 5, atrophic duodenal cells contained prominent iron deposits in the cytoplasm, which was more prominent in the TGs than in the WTs (Figure 5C). Quantitation showed approximately $680 \%$ more iron staining in the TGs (TGs, $384 \pm 78$ arbitrary units [AU]; WTs, $49 \pm 18$ $\mathrm{AU}$; means $\pm \mathrm{SEM}, N=5$ to $6, P<0.005$ ).

\section{Oxidative Stress in Duodenal Mucosal Cells of TGs Under an Iron-Rich Diet}

We evaluated the oxidative stress in the duodenal mucosal cells under an iron-rich diet at day 5. Significant increases in nuclear 8-OHdG and cytoplasmic HNE modified proteins were observed in the degenerated duodenal mucosal cells (Figure 5D). Quantification showed approximately $250 \%$ more $8-\mathrm{OHdG}$ in the TGs (TGs, $209 \pm$ $76 \mathrm{AU}$; WTs, $59 \pm 34 \mathrm{AU}$; means $\pm \mathrm{SEM}, N=4, P<0.05)$ and $180 \%$ more HNE-modified proteins in the TGs (TGs, $270 \pm 60 \mathrm{AU}$; WTs, $96 \pm 48 \mathrm{AU}$; means \pm SEM, $N=4$ to $5, P<0.05)$. These results demonstrated that the cause of cellular degeneration is oxidative stress via excess iron.

\section{Discussion}

Knocking out the function of SLC11A2 causes microcytic anemia resulting from iron shortage, as demonstrated in rodent models. ${ }^{9,10}$ To directly assess the pathological significance of SLC11A2 overexpression, we generated three independent lines of SLC11A2 TGs under the control of the $\beta$-actin promoter. Since insertional sites of transgene at least for two lines were different and the three lines presented similar phenotype, we concluded that the results were not due to insertional mutagenesis. SLC11A2 TGs were viable and fertile, developed normally and displayed no overt abnormalities with normal life span. The iron concentration in various organs of 18-week-old male TGs showed no significant differences from the corresponding wild-type littermates. However, in the duodenum of TGs, the iron absorption evaluated by ${ }^{55} \mathrm{Fe}$ was significantly higher than for wild-type mice.
Therefore, we confirmed that the transgene is functional in the TGs.

So far, SLC11A2 appears to migrate to a variety of molecular weights in the Western blot analysis. Investigators have reported different molecular weights ranging from 43 to $116 \mathrm{kDa}$ in different cells and species. ${ }^{24,29-33}$ This issue is probably associated with the following three points: firstly, SLC11A2 is a membrane protein with 12 membrane-spanning domains; secondly, there is a difference in the level of glycosylation in the different tissues; and thirdly, proteolysis either endogenous or during sample preparation is different. Membrane proteins are easily misfolded in vivo and aggregated during the sample preparation. In the present study, we have produced a polyclonal antibody and evaluated its specificity by many different methods. Dot blot of tail lysates were useful to select TGs and the immunohistochemical results were satisfactory. Thus, we believe that the specificity of this antibody is high. Development of the optimal protocol for Western blot analysis of each tissue and why different organs show different molecular weights requires further investigation.

What surprised us in the TGs was the lack of SLC11A2 expression in hepatocytes despite ample expression in Kupffer cells and bile ducts. Powerful mechanisms to block expression despite the presence of the $\beta$-actin promoter are at work. It is currently unknown and under investigation whether this is accomplished at the transcriptional level or posttranscriptional level, including miRNA, posttranscriptional mechanism, or protein degradation.

We tried to find a phenotypic difference between TGs and wild-type mice. We used an acute phase of ferric nitrilotriacetate-induced renal carcinogenesis mode $\left.\right|^{34,35}$ and iron depletion with an iron-deficient diet, and we evaluated oxidative stress and hematocrit with serum iron concentration as end-points. However, these attempts were not successful (T. Shirase, S. Toyokuni, unpublished data). The only difference that we detected was that there was oxidative stress in the intestinal mucosal cells after an excessively iron-fortified diet in the TGs.

Originally, we anticipated higher iron levels in a variety of organs, which might have caused a higher incidence of cancer. ${ }^{4}$ However, our hypothesis was hampered by what we refer to as a "mucosal block." ${ }^{36}$ Even under a basal diet, the expression levels of ferroportin-1, which is associated with iron efflux from the duodenal mucosal cells into circulation, ${ }^{37,38}$ and those of transferrin receptor were significantly suppressed in the TGs, so the iron regulatory mechanisms were stricter than we expected. It is of note that ferroportin-1 expression was suppressed independent of hepatic hepcidin. This suggests that regulation of ferroportin-1 in duodenal enterocytes occurs here through intracellular mechanisms, presumably through the intracellular iron content, and not necessarily through increased production of hepcidin. ${ }^{39}$

Under an iron-rich diet, the mice showed a marked increase in iron concentration in the liver and spleen with no difference between TGs and WTs, demonstrating that iron absorption mechanisms were functional. However, decreased expression of ferroportin-1, together with in- 
creased expression of hepcidin, which is a blocking ligand for ferroportin, ${ }^{39}$ was responsible for the iron-mediated oxidative stress in the duodenal mucosal cells of TGs. Therefore, SLC11A2 overexpression in the duodenum presents a risk for oxidative duodenal cell damage under a luminal iron-rich condition. Why iron-deficient diet did not induce merits for TG mice is unknown at present. We can propose two possibilities for this: (1) iron absorption via heme transporter was independently functional; (2) strict animal experiments were difficult, since we observed that mice ate their feces. The use of special animal containers would rule out this possibility.

Therefore, we concentrated on iron toxicity in the duodenum. Ferric citrate remains one of the most commonly used nutrition reinforcement. However, excess oral iron intake may cause iron poisoning. Accidental ingestion of these iron tablets and iron-containing vitamins by children less than 6 years of age still occurs today, and even fatal cases have been reported. ${ }^{40}$ This poisoning can cause bloody diarrhea and vomiting due to the damage of the intestinal epithelial linings. ${ }^{41,42}$ Erosive injury to the upper gastrointestinal tract in patients receiving iron medication has been reported as a side effect. ${ }^{43}$ In the present experiments, ferric citrate was easily absorbed, leading to extremely high levels of iron in the liver both in TGs and wild-type mice. Our observation suggests that iron fortification may lead to unexpected iron overload in some patients, and may aggravate the health condition. We also have to reconsider the population for iron fortification as a public health issue since iron excess is associated with carcinogenesis, ${ }^{3,4,44}$ and phlebotomy or blood donation decreased the risk for cancer ${ }^{45,46}$ despite the presence of some controversies.

In our TGs, excess iron influx with limited iron efflux caused iron to accumulate in the duodenal cells, which resulted in oxidative stress and hemorrhagic necrosis. Our results may have clinical implications. Firstly, SLC11A2 overexpression was associated with a worsened intestinal erosive inflammation, including differences in the severity and the anatomical areas involved. Single nucleotide polymorphisms are known to exist in this gene in humans. Thus, after evaluating the function of each genotype of SLC11A2, a prevention of side effects from iron tablets might be possible by adjusting the iron dose. Secondly, duodenal ulcer is a relatively common disease. Investigation of the association of this disease and SLC11A2 expression as well as single nucleotide polymorphisms may open up novel ways to better understand the pathology of this disease.

In conclusion, we created the first SLC11A2 transgenic mice under the control of the $\beta$-actin promoter. These mice presented with an apparently normal phenotype and normal life span, and they were found to be fertile. However, under an iron-rich diet, massive intestinal ulcers with oxidative stress occurred, which resulted from iron accumulation in the cytoplasm of the intestinal mucosal cells. Our findings suggest that duodenal mucosal cells work as two functional gates to prevent global iron overload and that finely regulated duodenal absorption is essential to protect intestinal mucosa from iron intoxication.

\section{References}

1. Wriggleworth JM, Baum H: The biochemical function of iron. Iron in biochemistry and medicine, II. Edited by A Jacobs, M Worwood. London, Academic Press, 1980, pp. 29-86

2. Dunn L, Rahmanto Y, Richardson D: Iron uptake and metabolism in the new millennium. Trends Cell Biol 2007, 17:93-100

3. Toyokuni S: Iron-induced carcinogenesis: the role of redox regulation Free Radic Biol Med 1996, 20:553-566

4. Toyokuni S: Role of iron in carcinogenesis: cancer as a ferrotoxic disease. Cancer Sci 2009, 100:9-16

5. Gunshin H, Mackenzie B, Berger U, Gunshin Y, Romero M, Boron W Nussberger S, Gollan J, Hediger M: Cloning and characterization of a mammalian proton-coupled metal-ion transporter. Nature 1997, 388:482-488

6. Canonne-Hergaux F, Gruenheid S, Ponka P, Gros P: Cellular and subcellular localization of the Nramp2 iron transporter in the intestinal brush border and regulation by dietary iron. Blood 1999, 93:4406-4417

7. Canonne-Hergaux F, Levy J, Fleming M, Montross L, Andrews N, Gros P: Expression of the DMT1 (NRAMP2/DCT1) iron transporter in mice with genetic iron overload disorders. Blood 2001, 97:1138-1140

8. Trinder D, Oates P, Thomas C, Sadleir J, Morgan E: Localisation of divalent metal transporter 1 (DMT1) to the microvillus membrane of rat duodenal enterocytes in iron deficiency, but to hepatocytes in iron overload. Gut 2000, 46:270-276

9. Fleming M, Trenor C, Su M, Foernzler D, Beier D, Dietrich W, Andrews $\mathrm{N}$ : Microcytic anaemia mice have a mutation in Nramp2, a candidate iron transporter gene. Nat Genet 1997, 16:383-386

10. Fleming M, Romano M, Su M, Garrick L, Garrick M, Andrews N: Nramp2 is mutated in the anemic Belgrade (b) rat: evidence of a role for Nramp2 in endosomal iron transport. Proc Natl Acad Sci USA 1998, 95:1148-1153

11. Mims M, Guan Y, Pospisilova D, Priwitzerova M, Indrak K, Ponka P, Divoky $\mathrm{V}$, Prchal J: Identification of a human mutation of DMT1 in a patient with microcytic anemia and iron overload. Blood 2005 105:1337-1342

12. Iolascon A, d'Apolito M, Servedio V, Cimmino F, Piga A, Camaschella C: Microcytic anemia and hepatic iron overload in a child with compound heterozygous mutations in DMT1 (SCL11A2). Blood 2006 107:349-354

13. Gunshin H, Fujiwara Y, Custodio A, Direnzo C, Robine S, Andrews N: Slc11a2 is required for intestinal iron absorption and erythropoiesis but dispensable in placenta and liver. J Clin Invest 2005, 115:1258-1266

14. Hubert N, Hentze M: Previously uncharacterized isoforms of divalent metal transporter (DMT)-1: implications for regulation and cellular function. Proc Natl Acad Sci USA 2002, 99:12345-12350

15. Tabuchi M, Tanaka N, Nishida-Kitayama J, Ohno H, Kishi F: Alternative splicing regulates the subcellular localization of divalent metal transporter 1 isoforms. Mol Biol Cell 2002, 13:4371-4387

16. Zhong $Y$, Jiang L, Hiai H, Toyokuni S, Yamada $Y$ : Overexpression of a transcription factor LYL1 induces T- and B-cell lymphoma in mice. Oncogene 2007, 26:6937-6947

17. Tsuruyama T, Nakamura T, Jin G, Ozeki M, Yamada Y, Hiai H: Constitutive activation of Stat5a by retrovirus integration in early pre-B lymphomas of SL/Kh strain mice. Proc Natl Acad Sci USA 2002, 99:8253-8258

18. Toyokuni S, Tanaka T, Hattori Y, Nishiyama Y, Ochi H, Hiai H, Uchida K, Osawa T: Quantitative immunohistochemical determination of 8-hydroxy-2'-deoxyguanosine by a monoclonal antibody N45.1: its application to ferric nitrilotriacetate-induced renal carcinogenesis model. Lab Invest 1997, 76:365-374

19. Toyokuni S, Miyake N, Hiai H, Hagiwara M, Kawakishi S, Osawa T, Uchida K: The monoclonal antibody specific for the 4-hydroxy-2nonenal histidine adduct. FEBS Lett 1995, 359:189-191

20. Toyokuni S, Akatsuka S: Pathological investigation of oxidative stress in the postgenomic era. Pathol Int 2007, 57:461-473

21. Tanaka T, Nishiyama Y, Okada K, Hirota K, Matsui M, Yodoi J, Hiai H, Toyokuni S: Induction and nuclear translocation of thioredoxin by oxidative damage in the mouse kidney: independence of tubular necrosis and sulfhydryl depletion. Lab Invest 1997, 77:145-155

22. Toyokuni S, Kawaguchi W, Akatsuka S, Hiroyasu M, Hiai H: Intermittent microwave irradiation facilitates antigen-antibody reaction in Western blot analysis. Pathol Int 2003, 53:259-261 
23. Dutta KK, Nishinaka Y, Masutani H, Akatsuka S, Aung TT, Shirase T, Lee W-H, Hiai H, Yodoi J, Toyokuni S: Two distinct mechanisms for loss of thioredoxin-biding protein-2 in oxidative stress-induced renal carcinogenesis. Lab Invest 2005, 85:798-807

24. Tabuchi M, Yoshimori T, Yamaguchi K, Yoshida T, Kishi F: Human NRAMP2/DMT1, which mediates iron transport across endosomal membranes, is localized to late endosomes and lysosomes in HEp-2 cells. J Biol Chem 2000, 275:22220-22228

25. Liu Y-T, Shang D-G, Akatsuka S, Ohara H, Dutta KK, Mizushima K, Naito Y, Yoshikawa T, Izumiya M, Abe K, Nakagama H, Noguchi N, Toyokuni S: Chronic oxidative stress causes amplification and overexpression of ptprz1 protein tyrosine phosphatase to activate $\beta$-catenin pathway. Am J Pathol 2007, 171:1978-1988

26. Drake S, Morgan E, Herbison C, Delima R, Graham R, Chua A, Leedman P, Fleming R, Bacon B, Olynyk J, Trinder D: Iron absorption and hepatic iron uptake are increased in a transferrin receptor 2 (Y245X) mutant mouse model of hemochromatosis type 3. Am J Physiol Gastrointest Liver Physiol 2007, 292:G323-G328

27. Dupic F, Fruchon S, Bensaid M, Loreal O, Brissot P, Borot N, Roth M, Coppin $\mathrm{H}$ : Duodenal mRNA expression of iron related genes in response to iron loading and iron deficiency in four strains of mice. Gut 2002, 51:648-653

28. Toyokuni S, Okada S, Hamazaki S, Fujioka M, Li J-L, Midorikawa O: Cirrhosis of the liver induced by cupric nitrilotriacetate in Wistar rats: an experimetnal model of copper toxicosis. Am J Pathol 1989, 134:1263-1274

29. Conrad M, Umbreit J, Moore E, Hainsworth L, Porubcin M, Simovich M, Nakada M, Dolan K, Garrick M: Separate pathways for cellular uptake of ferric and ferrous iron. Am J Physiol Gastrointest Liver Physiol 2000, 279:G767-G774

30. Canonne-Hergaux F, Fleming M, Levy J, Gauthier S, Ralph T, Picard $V$, Andrews N, Gros P: The Nramp2/DMT1 iron transporter is induced in the duodenum of microcytic anemia mk mice but is not properly targeted to the intestinal brush border. Blood 2000, 96:3964-3970

31. Yeh K, Yeh M, Watkins J, Rodriguez-Paris J, Glass J: Dietary iron induces rapid changes in rat intestinal divalent metal transporter expression. Am J Physiol Gastrointest Liver Physiol 2000, 279:G1070-G1079

32. Canonne-Hergaux F, Zhang A, Ponka P, Gros P: Characterization of the iron transporter DMT1 (NRAMP2/DCT1) in red blood cells of normal and anemic mk/mk mice. Blood 2001, 98:3823-3830

33. Roth J, Horbinski C, Feng L, Dolan K, Higgins D, Garrick M: Differential localization of divalent metal transporter 1 with and without iron response element in rat PC12 and sympathetic neuronal cells. J Neurosci 2000, 20:7595-7601

34. Li JL, Okada S, Hamazaki S, Ebina Y, Midorikawa O: Subacute nephrotoxicity and induction of renal cell carcinoma in mice treated with ferric nitrilotriacetate. Cancer Res 1987, 47:1867-1869

35. Toyokuni S, Okada S, Hamazaki S, Minamiyama Y, Yamada Y, Liang $\mathrm{P}$, Fukunaga $\mathrm{Y}$, Midorikawa $\mathrm{O}$ : Combined histochemical and biochemical analysis of sex hormone dependence of ferric nitrilotriacetate-induced renal lipid peroxidation in ddY mice. Cancer Res 1990, 50:5574-5580

36. Nathanson M, McLaren G, Saidel G: A model of intestinal iron absorption and plasma iron kinetics: optimal parameter estimates for normal dogs. Comput Biomed Res 1984, 17:55-70

37. McKie A, Marciani P, Rolfs A, Brennan K, Wehr K, Barrow D, Miret S, Bomford A, Peters T, Farzaneh F, Hediger M, Hentze M, Simpson R: A novel duodenal iron-regulated transporter, IREG1, implicated in the basolateral transfer of iron to the circulation. Mol Cell 2000, 5:299-309

38. Montosi G, Donovan A, Totaro A, Garuti C, Pignatti E, Cassanelli S, Trenor C, Gasparini P, Andrews N, Pietrangelo A: Autosomal-dominant hemochromatosis is associated with a mutation in the ferroportin (SLC11A3) gene. J Clin Invest 2001, 108:619-623

39. Nemeth E, Preza G, Jung C, Kaplan J, Waring A, Ganz T: The $\mathrm{N}$-terminus of hepcidin is essential for its interaction with ferroportin: structure-function study. Blood 2006, 107:328-333

40. Litovitz T, Klein-Schwartz W, White S, Cobaugh D, Youniss J, Drab A, Benson B: 1999 annual report of the American Association of Poison Control Centers Toxic Exposure Surveillance System. Am J Emerg Med 2000, 18:517-574

41. Tenenbein M, Littman C, Stimpson R: Gastrointestinal pathology in adult iron overdose. J Toxicol Clin Toxicol 1990, 28:311-320

42. Products TEAftEoM: Committee for Veterinary Medicinal Products Iron Dichloride and Iron Sulfate: Summary Report. London, UK, 1998, pp. 1-3

43. Abraham S, Yardley J, Wu T: Erosive injury to the upper gastrointestinal tract in patients receiving iron medication: an under-recognized entity. Am J Surg Pathol 1999, 23:1241-1247

44. Hercberg S, Estaquio C, Czernichow S, Mennen L, Noisette N Bertrais S, Renversez J, Briancon S, Favier A, Galan P: Iron status and risk of cancers in the SU.VI.MAX cohort. J Nutr 2005, 135:2664-2668

45. Zacharski L, Chow B, Howes P, Shamayeva G, Baron J, Dalman R, Malenka D, Ozaki C, Lavori P: Reduction of iron stores and cardiovascular outcomes in patients with peripheral arterial disease: a randomized controlled trial. JAMA 2007, 297:603-610

46. Edgren G, Reilly M, Hjalgrim H, Tran T, Rostgaard K, Adami J, Titlestad K, Shanwell A, Melbye M, Nyren O: Donation frequency, iron loss, and risk of cancer among blood donors. J Natl Cancer Inst 2008, 100:572-579 